\title{
Abnormalities in Hippocampal Functioning with Persistent Pain
}

\author{
Amelia A. Mutso, ${ }^{1}$ Daniel Radzicki, ${ }^{1}$ Marwan N. Baliki, ${ }^{1}$ Lejian Huang, ${ }^{1}$ Ghazal Banisadr, ${ }^{2}$ Maria V. Centeno, ${ }^{1}$ \\ Jelena Radulovic, ${ }^{3}$ Marco Martina, ${ }^{1}$ Richard J. Miller, ${ }^{2}$ and A. Vania Apkarian ${ }^{1,4}$ \\ Departments of ${ }^{1}$ Physiology, ${ }^{2}$ Molecular Pharmacology and Biological Chemistry, ${ }^{3}$ Psychiatry and Behavioral Sciences, and ${ }^{4}$ Anesthesia and Surgery, \\ Feinberg School of Medicine, Northwestern University, Chicago, Illinois 60611
}

Chronic pain patients exhibit increased anxiety, depression, and deficits in learning and memory. Yet how persistent pain affects the key brain area regulating these behaviors, the hippocampus, has remained minimally explored. In this study we investigated the impact of spared nerve injury (SNI) neuropathic pain in mice on hippocampal-dependent behavior and underlying cellular and molecular changes. In parallel, we measured the hippocampal volume of three groups of chronic pain patients. We found that SNI animals were unable to extinguish contextual fear and showed increased anxiety-like behavior. Additionally, SNI mice compared with Sham animals exhibited hippocampal (1) reduced extracellular signal-regulated kinase expression and phosphorylation, (2) decreased neurogenesis, and (3) altered short-term synaptic plasticity. To relate the observed hippocampal abnormalities with human chronic pain, we measured the volume of human hippocampus in chronic back pain (CBP), complex regional pain syndrome (CRPS), and osteoarthritis patients (OA). Compared with controls, CBP and CRPS, but not OA, had significantly less bilateral hippocampal volume. These results indicate that hippocampus-mediated behavior, synaptic plasticity, and neurogenesis are abnormal in neuropathic rodents. The changes may be related to the reduction in hippocampal volume we see in chronic pain patients, and these abnormalities may underlie learning and emotional deficits commonly observed in such patients.

\section{Introduction}

Recent work in both human patients and laboratory animals has demonstrated that the presence of chronic neuropathic pain causes gross reorganization and functional changes in both cortical and subcortical structures, including the medial prefrontal cortex (Baliki et al., 2006, 2008; Metz et al., 2009), thalamus (Apkarian et al., 2004a), amygdala (Han and Neugebauer, 2005; Ji et al., 2010), and anterior cingulate cortex (Li et al., 2010). These areas are associated with functions including learning, memory, fear, and emotional responses and map on to cognitive and emotional problems suffered by chronic pain patients, such as elevated anxiety and depression (Gore et al., 2011), problems in emotional decision making (Apkarian et al., 2004b), working memory (Dick and Rashiq, 2007), and difficulty in classical conditioning tasks (Flor et al., 2002). However, the hippocampus, which is one of the crucial limbic areas involved in learning and memory with which many of the above brain areas interact, has been studied less systematically, especially in regards to the impact of chronic pain on its function.

Received Feb. 7, 2012; revised March 6, 2012; accepted March 12, 2012.

Author contributions: A.A.M., D.R., M.N.B., L.H., G.B., J.R., M.M., R.J.M., and A.V.A. designed research; A.A.M., D.R., M.N.B., L.H., G.B., and M.V.C. performed research; A.A.M., D.R., M.N.B., L.H., G.B., and M.M. analyzed data; A.A.M., M.M., and A.V.A. wrote the paper.

This study was supported by National Institutes of Health (NH)-National Institute of Neurological Disorders and Stroke Grants NS57704, NS5064091, NIH-National Institute of Mental Health MH73669, and NIH T32 AG020418 - 09.

Correspondence should be addressed to A. Vania Apkarian at the above address. E-mail: a-apkarian@northwestern.edu.

DOI:10.1523/JNEUROSCI.0587-12.2012

Copyright $\odot 2012$ the authors $\quad 0270-6474 / 12 / 325747-10 \$ 15.00 / 0$
Recent studies have independently shown hippocampal abnormalities in animal models of chronic pain including shortterm (Ren et al., 2011) and recognition memory deficits (Kodama et al., 2011), abnormal cytokine expression (Al-Amin et al., 2011; del Rey et al., 2011), deficits in long-term potentiation (Kodama et al., 2007), and impaired enriched-environment neurogenesis (Terada et al., 2008). Additionally, a human patient study suggests reduced hippocampal volume in elderly individuals with chronic pain (Zimmerman et al., 2009). Here we offer a comprehensive within-subject approach to define the cognitive and emotional role of the hippocampus in chronic pain by specifically examining hippocampal-dependent contextual fear conditioning and extinction, anxiety-like behavior, hippocampal expression of a protein kinase linked to contextual extinction, hippocampal neurogenesis, and short-term hippocampal synaptic plasticity through the use of spared nerve injury (SNI) mice. Additionally we assess human hippocampal volume in three clinical chronic pain conditions.

The hippocampus is critically involved in anxiety, depression, learning, and memory (Barkus et al., 2010) and is essential in contextual conditioning and extinction (Phillips and LeDoux, 1992). We hypothesize that chronic pain can be redefined in terms of context conditioning and extinction, and viewed as a state of continual learning through which aversive emotional associations are constantly made with otherwise incidental events due to the persistent presence of a patient's pain. Moreover, due to the constant presence of the pain, such chronic pain patients may become unable to extinguish the conditioned event since at each re-exposure pain may still be present (Apkarian, 2008; 
Apkarian et al., 2009, 2011). Within this context, the hippocampus, due to its specific role in contextual learning, may become dysregulated. In fact, our results indicate a disruption of hippocampal-dependent contextual extinction learning accompanied by a cascade of related hippocampal abnormalities. Relatedly, we also observed a decrease in hippocampal volume in human patients with chronic pain, which may be associated with the behavioral and cellular events we observe in mice.

\section{Materials and Methods}

\section{Animals}

\section{Experiments 1 and 2}

C57BL/6 male mice were obtained at 9 weeks of age from Harlan (Experiment 1). Double cortin-enhanced green fluorescence protein (DCXEGFP) transgenic mice were used for Experiment 2. The mice were housed individually with a $12 \mathrm{~h}$ light/dark cycle (7 A.M.-7 P.M.) with food and water available ad libitum. All studies were approved by the Animal Care and Use Committee of Northwestern University. The mice were operated on at 9 weeks of age and tactile thresholds were measured before surgical manipulation and again on days $3,7,9$, and 14 postsurgery to ensure surgical success (except in the Fear Conditioning group where measurements were taken at day 27 instead of 14 in order to not interfere with the experiment).

\section{Experiment 3}

C57BL/6 male mice were obtained from Harlan and operated on at p21. Mice were housed with littermates under the same conditions as above. Tactile thresholds were measured before surgical manipulation and again on day 7 postsurgery before killing the animal.

\section{Neuropathic pain model: SNI}

The SNI model has been described previously (Decosterd and Woolf, 2000). Mice were anesthetized with isoflurane $1.5-2 \%$ and a mixture of $30 \% \mathrm{~N}_{2} \mathrm{O}$ and $70 \% \mathrm{O}_{2}$. One hindleg sciatic nerve (varied over different experiments) was exposed at the level of the trifurcation into the sural, tibial, and common peroneal nerves. The tibial and common peroneal nerves were tightly ligated and severed, leaving the sural nerve intact. Animals in the Sham surgery group had their sciatic nerve exposed as in the SNI procedure but received no further manipulation.

\section{Tactile sensitivity}

Paw withdrawal thresholds to von Frey filament stimulation (VF) were used to assess mechanical sensitivity of the hindpaws. Animals were placed in a Plexiglas box with a wire grid floor and allowed to habituate for $15 \mathrm{~min}$. At this point, filaments of various forces (Stoelting) were applied to the plantar surface of each hindpaw. Filaments were applied in a descending or ascending pattern, determined by the response of the animal. Each filament was applied for a maximum of $2 \mathrm{~s}$, and paw withdrawal in response to the filament was considered a positive response. Fifty percent thresholds were calculated according to Chaplan et al. (1994). To be entered into the SNI testing group, animals undergoing SNI surgery were required to decrease their injured paw withdrawal latency significantly when compared with the control paw and Sham animals.

\section{Experiment 1: Hippocampus-mediated behavior and corresponding protein kinase activity}

\section{Fear conditioning and extinction}

Fear conditioning was performed on a subgroup of mice (SNI, $n=11$; Sham, $n=10$; left-side surgery) on postoperative day 10 with an automated system (TSE) and consisted of a single exposure to context ( $3 \mathrm{~min}$ ) followed by a $2 \mathrm{~s}$ tone paired with foot shock $(2 \mathrm{~s}, 0.7 \mathrm{~mA}$, constant current) as described previously (Radulovic et al., 1999). The extinction trials were performed at $24 \mathrm{~h}$ intervals and consisted of nonreinforced 3 min exposures to the context (Fischer et al., 2007). Context-dependent freezing was measured every fifth second over 3 min and expressed as percentage of the total number of observations. Mice were followed until Sham animals showed significant extinction. At this time mice were placed in a different conditioning box where the previously used tone was presented for $3 \mathrm{~min}$. These tone extinction trials were performed at $24 \mathrm{~h}$ intervals, and tone-dependent freezing was measured every fifth second over 3 min.

\section{Anxiety behavioral testing}

Anxiety behavioral testing was performed on a subgroup of mice (SNI, $n=22,11$ left-side surgery, 11 right-side surgery; Sham, $n=20,10$ left-side surgery, 10 right-side surgery). Experiments began on postoperative day 12. These mice did not undergo fear conditioning or extinction.

Black box emergence. This test is used as a measure of anxiety-like behavior, in that it measures fear of exposure to an unfamiliar situation. Animals prefer small enclosed chambers on their initial exposure to environments, and if the environment is made more unfamiliar or aversive they spend more time in the small enclosure before exploring. If the animal is more anxious, it will spend a longer time in the chamber before emerging into an open testing space (Takahashi et al., 1989). A threesided covered box was placed in the center of an open field illuminated by bright light (to increase open-field aversiveness). The behavior of the mice was recorded by a video camera connected to a computer and analyzed by TSE software (VideoMot). Mice were placed in the dark box and the latency for them to leave the box was recorded. The latency to leave the black box was interpreted as anxiety-like behavior (Kishimoto et al., 2000).

Novelty suppressed feeding. In this test there are competing motivations between the drive to eat and fear of entering a brightly lit center area. Food was removed from the home cage $24 \mathrm{~h}$ before testing and water was removed $2 \mathrm{~h}$ before testing. One pellet of food was placed in the center of an open field and mice were placed in one corner of the open field. The behavior of the mice was recorded by a video camera connected to a computer and analyzed by TSE software (VideoMot). The behavior of the mice was recorded for $5 \mathrm{~min}$ and the latency to start eating was recorded. Increased latency was interpreted as an increased anxiety, since classical anxiolytic drugs and selective serotonin reuptake inhibitor treatment decrease this behavior (David et al., 2009). Due to the latency being capped at $5 \mathrm{~min}$, a Mann-Whitney $U$ test was used in the analysis. This test was adapted from Santarelli et al. (2003).

\section{Shock reactivity}

Shock reactivity was measured 2 weeks postextinction on the same subgroup of mice that underwent fear conditioning and extinction. The technique consisted of three sets with each set given $24 \mathrm{~h}$ apart (Turner et al., 1967) in the same conditioning box as used for cue extinction. A set consisted of a series of eight shock intensities ( $\mathrm{mA}$, constant current): 0.1, $0.2,0.3,0.4,0.5,0.6,0.7$, and 0.8 . Shocks were given in ascending order with a $10 \mathrm{~s}$ interval between shocks. Following each shock, the reaction of the mouse was recorded as no response, flinch, jump, or vocalize. The intensity at which the mouse first responded with a flinch, jump, or vocalization was recorded.

\section{Protein levels analysis}

Tissue was collected from a subgroup of mice which had not undergone fear conditioning or extinction (SNI, $n=6$; Sham, $n=5$; right-side surgery) for Western blot analysis. Both right and left dorsal and ventral hippocampus were collected. Hippocampal lysates were subject to $4-12 \%$ Bis-Tris Gel (Invitrogen) electrophoresis, and subsequently blotted to nitrocellulose membranes (Bio-Rad). Membranes saturated with block were incubated with anti-phos-extracellular signal-regulated kinase (ERK) (dpERK; 1:250, Sigma-Aldrich), anti-ERK (1:500; Santa Cruz Biotechnology), and anti- $\beta$-tubulin (1:500; Sigma-Aldrich). Western blots were developed under maximal chemiluminescence emission with Western-Lighting Plus-ECl (PerkinElmer) on Bio-Rad QuantityOne. pERK and ERK were normalized to $\beta$-tubulin levels independently to account for ERK expression differences.

\section{Experiment 2: Hippocampal neurogenesis}

Bromodeoxyuridine labeling

Bromodeoxyuridine (BrdU) was used to evaluate cell proliferation. DCX-EGFP mice (SNI, $n=5$; Sham, $n=6$; left-side surgery) received 
intraperitoneal injections of BrdU (Sigma-Aldrich) $50 \mathrm{mg} / \mathrm{kg}$ dissolved in sterile PBS $(10 \mathrm{mg} / \mathrm{ml})$ and filtered. Injections were given starting day 3 postsurgery (SNI or Sham) and continued for $5 \mathrm{~d}$. Mice were perfused 1 week after the last injection ( $14 \mathrm{~d}$ postsurgery).

\section{Immunohistochemistry}

Mice were transcardially perfused with ice-cold $4 \%$ paraformaldehyde (PFA) in phosphate buffer ( $\mathrm{pH} 7.4,150 \mathrm{ml} /$ mouse). Brains were postfixed for $48 \mathrm{~h}$ in $4 \%$ PFA and sections were cut on a vibratome (Leica VT 1000S; Leica Microsystems) and collected in cold PBS. Free-floating sections were then incubated with primary antibodies for $\operatorname{BrdU}(1: 500$; Fitzgerald) and NeuN (1:200; Millipore), a marker of mature neurons, to allow for triple-illumination of DCX, BrdU, and NeuN. Following primary incubation, the corresponding secondary antibody conjugated with Alexa Fluor 633 (Invitrogen) was used for NeuN and biotinconjugated secondary (Jackson ImmunoResearch) along with streptavidin Alexia 546 (Jackson ImmunoResearch), which was used for BrdU. Slices were mounted in Vectashield (Vector) and observed with a fluorescence microscope (Olympus Fluoview FV10i, confocal laser scanning microscope) for triple-labeling at $60 \times$. Two consecutive hippocampal sections per mouse were live counted for double- and triple-labeling in a blinded fashion (bilateral). Images were taken through the microscope.

\section{Experiment 3: Hippocampal dentate gyrus-CA3 fEPSP potentiation}

Preparation of hippocampal slices

Mice (SNI, $n=13$; Sham, $n=12$; left-side surgery) were anesthetized using isoflurane and killed by decapitation at day 7 postsurgery. The brain was quickly removed and placed in ice-cold sucrose-rich artificial CSF (ACSF) containing the following (in $\mathrm{mm}$ ): $87 \mathrm{NaCl}, 25 \mathrm{NaHCO}_{3}, 25$ glucose, 75 sucrose, $2.5 \mathrm{KCl}, 1.25 \mathrm{NaH}_{2} \mathrm{PO}_{4}, 0.5 \mathrm{CaCl}_{2}, 7 \mathrm{MgCl}_{2}, 1$ sodium pyruvate, and $5 \mu \mathrm{m}$ glutathione, and $400 \mu \mathrm{m}$ horizontal slices of the contralateral (SNI, $n=6$; Sham, $n=5$ ) or ipsilateral (SNI, $n=7$; Sham, $n=7$ ) hippocampus (relative to the peripheral nerve injury) were cut in the ventral to dorsal direction using a tissue slicer (Thermo Scientific). Slices were then stored in ACSF containing the following (in $\mathrm{mM}$ ): 125 $\mathrm{NaCl}, 25 \mathrm{NaHCO}_{3}, 25$ glucose, $2.5 \mathrm{KCl}, 1.25 \mathrm{NaH}_{2} \mathrm{PO}_{4}, 2 \mathrm{CaCl}_{2}, 1 \mathrm{MgCl}_{2}$, 1 sodium pyruvate, and $5 \mu \mathrm{M}$ glutathione for $20 \mathrm{~min}$ at $\sim 30^{\circ} \mathrm{C}$, followed by $20 \mathrm{~min}$ at $\sim 36^{\circ} \mathrm{C}$.

\section{Electrophysiological recordings}

Recordings were conducted in a chamber that was continuously perfused with ACSF containing $2.5 \mathrm{mM} \mathrm{CaCl}_{2}, 1 \mathrm{~mm} \mathrm{MgCl}, 100 \mu \mathrm{M}$ Picrotoxin (Ascent Scientific), and $50 \mu \mathrm{M}$ D-APV (Ascent Scientific). The hippocampus was visualized using differential interference contract infrared microscopy. A bipolar stimulating electrode (FHC) was positioned in the hilar region of the dentate gyrus. Glass (SG16; Dagan) recording electrodes containing $3 \mathrm{M} \mathrm{NaCl}$ and having tip resistances of $1-3 \mathrm{M} \Omega$ were placed in stratum lucidum of the CA3 region.

Electrophysiological recordings were performed at $31^{\circ} \mathrm{C} \pm 1$ using an Axopatch 200B amplifier and the stimulation was delivered by way of an A360 Stimulus Isolator (WPI). Field potential recordings were sampled at $10 \mathrm{kHz}$ and low-pass filtered at $5 \mathrm{kHz}$. Data were digitalized using a Digidata 1300 interface and acquired on a PC for off-line analysis.

The potentiation induction protocol was as follows: current stimulation levels were set to elicit excitatory field potentials of $40-60 \%$ of the maximum observed response. Baseline recordings were comprised of one $200 \mu$ s pulse every $15 \mathrm{~s}(0.067 \mathrm{~Hz})$ for $10 \mathrm{~min}$. Tetanic stimulation consisted of four $1 \mathrm{~s} 100 \mathrm{~Hz}$ trains at $30 \mathrm{~s}$ intervals. Post-tetanic potentiation (PTP) was calculated as the averaged slope of the first two responses following tetanic stimulation relative to control (average response in the 10 min preceding the tetanus).

\section{Experiment 4: Hippocampal volume of human chronic neuropathic pain patients \\ Subjects}

Fifty healthy volunteers (22 males, 28 females, mean age \pm SD: $40.1 \pm$ 11.3), 38 chronic back pain (CBP) patients (21 males, 17 females, mean age $\pm \mathrm{SD}: 48.4 \pm 9.7), 30$ complex regional pain syndrome (CRPS) patients ( 4 males, 26 females, mean age \pm SD: $41.5 \pm 11.6$ ), and 20 knee osteoarthritis (OA) patients ( 16 males, 4 females, mean age \pm SD: $53.1 \pm$ 7.5 ) were recruited for this study. All were right-handed and gave full informed consent to procedures approved by the Northwestern University International Review Board committee. These subjects are also included in the analysis of Baliki et al. (2011).

\section{Scanning parameters}

All high-resolution T1-anatomical brain images were scanned on a Siemens 3.0 T whole-body system with an eight-channel head coil and MPRAGE type image using the following parameters: voxel size $=1 \times$ $1 \times 1 \mathrm{~mm}, \mathrm{TR}=2500 \mathrm{~ms}, \mathrm{TE}=3.36 \mathrm{~ms}$, flip angle $=90^{\circ}$, in-plane matrix resolution $=256 \times 256$, slices $=160$ and field-of-view $=256 \mathrm{~mm}$.

\section{Data analyses}

Data were analyzed using the FSL 4.1 (Smith et al., 2004). First, the skull for each T1 image was extracted and the hippocampus was then segmented using FMRIB's integrated registration and segmentation tool (FIRST; Patenaude, 2007). After excluding those subjects who had inaccurate hippocampus segmentation (see details below), the volumes of right and left hippocampi were calculated for each subject. Finally, the group analyses of right and left hippocampus volumes, individually, were achieved after removing the effect of whole-brain volume, gender, and age as covariates of no interest.

\section{Segmentation results assessment}

We developed an objective procedure to assess the reliability of segmentation and, consequently, excluded those subjects that have unreliable segmentation results. This was a three-step procedure. First, to achieve a gross alignment of $\mathrm{T} 1$ images, the $\mathrm{T} 1$ image of each subject was initially coregistered to the MNI152 standard template by a rigid body transformation. Following this, 15 subjects' images from each group were randomly selected, averaged, and merged as a new template for each group, from which hippocampi were segmented as a target. Using the transformation matrix from the above coregistration, the hippocampus in subject space was transformed into standard space. Subjects whose standard hippocampus volume was outside of its group mean volume $\pm 1.0 \mathrm{SD}$ were considered unreliable and were not included in the analysis. Five subjects were excluded with this method.

\section{Results}

Hippocampus-mediated behavior and corresponding protein kinase activity

While both cue and context fear conditioning and extinction involve amygdala circuitry, contextual fear conditioning and extinction is unique in its involvement of the hippocampus (Phillips and LeDoux, 1992). Due to our hypothesis that chronic pain is associated with dysregulation of contextual learning, we first examined whether there is a difference in context and cue acquisition and extinction in a mouse model of neuropathic pain to determine whether there is a specific hippocampus conditioned learning deficit in contrast to the broader fear conditioning circuitry.

All animals demonstrated similar fear conditioned acquisition on testing day 1 (SNI, $n=11$; Sham, $n=10$ ). A significant difference was seen in context extinction $\left(F_{(19,190)}=3.6, p<\right.$ 0.001 ) with SNI animals showing an inability to extinguish to context. These animals showed no reduction in freezing behavior and continued to freeze $40 \%$ of observed time through extinction day 11. In contrast, Sham animals continued to diminish their freezing behavior over 11 extinction days to levels $<20 \%$ of observed time (Fig. 1a). In cue fear extinction, however, there was no difference between groups and both groups showed successful extinction in 3-4 d $\left(F_{(19,57)}=2.02\right.$, n.s.) (Fig. $\left.1 b\right)$. An illustration of the animal's mechanical thresholds can be seen in Figure $1 c$, which demonstrates that SNI mice (mean $=0.02 \mathrm{~g}$ ) exhibit mechanical allodynia on the side of nerve injury while Sham animals 
do not $\left(\right.$ mean $=0.44 \mathrm{~g}, t_{(19)}=5.935$, $p<0.001$ between groups postsurgery). SNI and Sham animals do not significantly differ from each other regarding shock intensity that first elicits flinching (SNI, mean $=0.13 \mathrm{~mA}$; Sham, mean $=$ $\left.0.14 \mathrm{~mA}, t_{(16)}=0.46\right)$, jumping (SNI, mean $=0.25 \mathrm{~mA}$; Sham, mean $=$ $\left.0.26 \mathrm{~mA}, t_{(19)}=0.18\right)$, and vocalizing $(\mathrm{SNI}$, mean $=0.35 \mathrm{~mA}$; Sham, mean $=$ $0.36 \mathrm{~mA}, t_{(19)}=0.18$ ) (Fig. $1 d$ ), and no differences were seen for these measures between trial days. This illustrates that the shock stimulation, inducing fear acquisition, was not confounded by the difference in mechanical allodynia between groups. Together, these results indicate that chronic neuropathic pain diminishes hippocampusmediated fear extinction behavior without affecting a hippocampalindependent form of fear extinction (cue extinction).

Latency to emerge from the black box was compared between SNI and Sham animals and a significant difference was seen between the two groups $\left(t_{(39)}=3.57, p=0.001\right)$ with SNI animals having a longer latency (mean $=$ $16.41 \mathrm{~s}$ ) than Sham animals (mean $=$ $2.00 \mathrm{~s}$ ) to leave the dark box (SNI, $n=21$; Sham, $n=20$ ) (Fig. $2 a$ ). SNI animals also had a prolonged latency until eating $($ mean $=271.09 \mathrm{~s}$ ) in the Novelty Suppressed Feeding task compared with Sham animals (mean $=224.95$; Mann-Whitney $U$ test, $U=129, Z=2.28, p=$ 0.02 ) (Fig. 2a). This demonstrates that SNI animals show evidence of hippocampal-dependent anxiety-like behaviors. However, this anxiety cannot be considered the cause of the deficit in context extinction since cue extinction is also diminished in studies of anxiety disorders (Green et al., 2011). Mechanical thresholds for these animals, also used for ERK analysis, are shown in Figure $2 b$. As with all SNI and Sham groups, SNI animals have significantly lower VF thresholds compared with Sham animals on the injured paw at every time point post surgery $\left(F_{(1,144)}=\right.$ 89.51, $p<0.001 ; \mathrm{d} 3$ : SNI, mean $=0.03 \mathrm{~g}$, Sham, mean $=0.41 \mathrm{~g}$, $t_{(19)}=4.82, p<0.001 ; \mathrm{d} 7: \mathrm{SNI}$, mean $=0.02 \mathrm{~g}$, Sham, mean $=$ $0.43 \mathrm{~g}, t_{(19)}=5.25, p<0.001 ; \mathrm{d} 9$ : SNI, mean $=0.03$, Sham, mean $=0.50, t_{(19)}=4.04, p<0.001 ; \mathrm{d} 14$ : SNI, mean $=0.02$, Sham, mean $\left.=0.61, t_{(19)}=4.60, p<0.001\right)$ (Fig. $\left.2 b\right)$. The uninjured paw shows no decrease in threshold in either group (Fig. 2b).

Previous research has revealed that ERK1/2 phosphorylation in the hippocampus is critical for contextual fear extinction (Fischer et al., 2007; Tronson et al., 2008), consequently, we examined basal levels of pERK and ERK in nonfear-conditioned SNI and Sham animals. In the ipsilateral to injury dorsal hippocampus, SNI animals showed a significant reduction in both pERK2: $\beta$-tubulin $\left(t_{(9)}=4.44, p=0.002\right)$ and ERK2: $\beta$-tubulin $\left(t_{(9)}=\right.$ 3.73, $p=0.004)$ compared with Sham animals (Fig. $3 a$ and $3 b$ ). Due to the decrease seen in ERK2 expression levels, the pERK2 expression difference can be attributed to a reduction in the total ERK2 gene expression. In contrast, in the ipsilateral ventral hippocampus, SNI animals show a significant reduction in pERK

Figure 1. Hippocampus-mediated behavior significantly modulated by chronic neuropathic pain. $\boldsymbol{a}$, Context fear extinction over 11 列 $\delta\left(t_{(19)}=2.44, p=0.02\right)$. Error bars indicate SEM, ${ }^{*} p<0.05 . \boldsymbol{b}$, Cue fear extinction over 4 trial days. Data presented are percentage paw presurgery or postsurgery in the healthy paw. $\boldsymbol{d}$, Shock reactivity of SNI and Sham animals across shock intensities. Groups did not differ at which stimulus intensity they first flinched $\left(t_{(16)}=0.45\right.$, n.s.), jumped $\left(t_{(19)}=0.18\right.$, n.s. $)$, or vocalized $\left(t_{(19)}=0.18\right.$, n.s.).
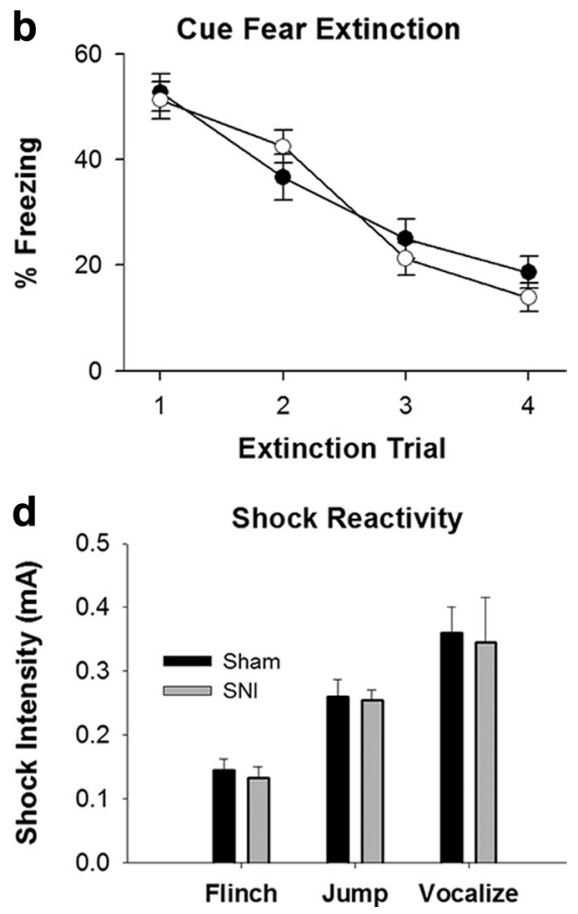
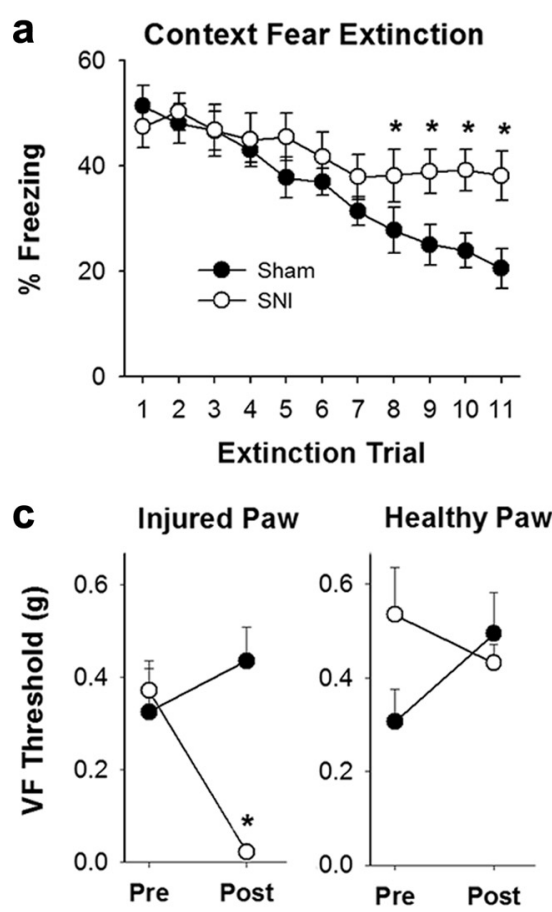

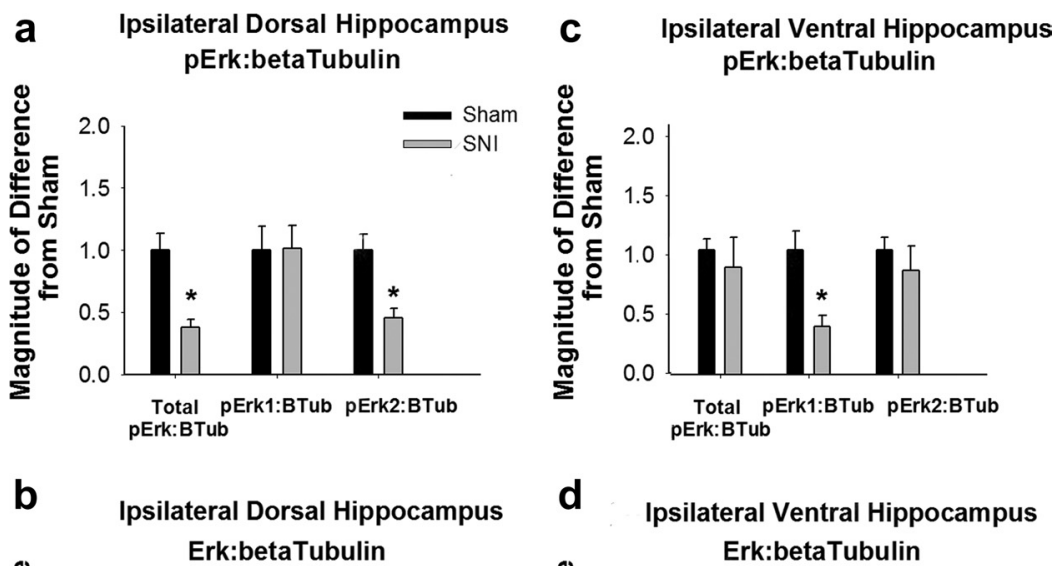

d
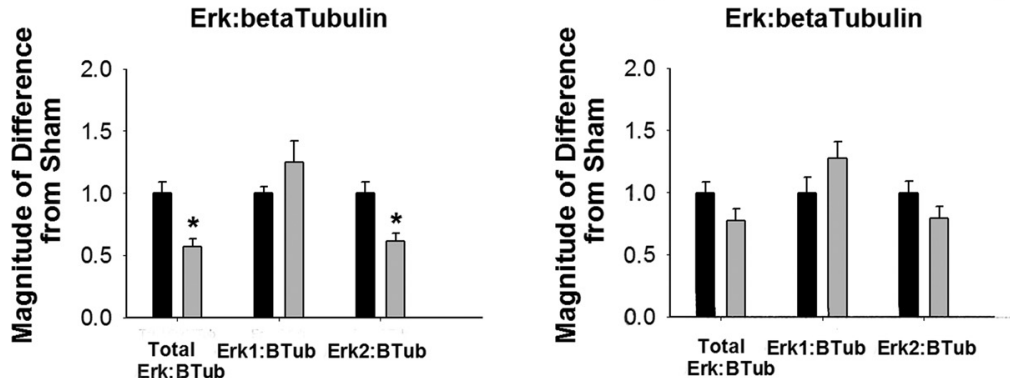

\begin{tabular}{|c|c|c|}
\hline & Ipsi Dorsal Hipp & Ipsi Ventral Hipp \\
\hline & Sham & Sham \\
\hline pErk & & \\
\hline Erk & and & \\
\hline BTubulin & Nines seves & \\
\hline
\end{tabular}

Figure 3. Ipsilateral hippocampal ERK expression and activity changes in SNI animals. a, pERK expression normalized to $\beta$-Tubulin in ipsilateral dorsal hippocampus. Data are normalized to Sham animal values, error bars indicate SEM, ${ }^{*} p<0.05$. SNI animals showed a significant reduction in $\mathrm{pERK} 2: \beta$-tubulin $\left(t_{(9)}=4.44, p=0.002\right)$ compared with Sham animals. No significant differences were seen in pERK1 activity. $\boldsymbol{b}$, ERK expression in ipsilateral dorsal hippocampus. SNI animals showed a significant reduction in ERK2: $\beta$ tubulin expression $\left(t_{(9)}=3.73, p=0.004\right)$, explaining the difference seen in $\boldsymbol{a}$ as a reduction in ERK2 expression in ipsilateral dorsal hippocampus of SNI animals. No significant differences were seen in ERK1 expression. $c$, pERK expression normalized to $\beta$-tubulin in ipsilateral ventral hippocampus. SNI animals showed a significant reduction in $\mathrm{pERK} 1: \beta$-tubulin expression compared with Sham animals $\left(t_{(9)}=3.68, p=0.005\right)$, while no reduction was seen in pERK2 activity. $\boldsymbol{d}$, ERK expression in ipsilateral ventral hippocampus. No significant differences were seen between groups. Table, Western blots for pERK, ERK, and $\beta$-tubulin in the hippocampus of SNI and Sham animals. pERK1 and ERK1 are the upper bands and pERK2 and ERK2 are the lower bands.

expression $\left(t_{(9)}=3.68, p=0.005\right)$, compared with Sham animals (Fig. 3c). No significant differences were seen in pERK2 expression or ERK expression (Fig. 3d). Due to the lack of changes in ERK expression, the difference in pERK1 expression in the ipsilateral ventral hippocampus is due to differences in ERK activity and phosphorylation, and not due to changes in gene expression.

In the contralateral hippocampus to injury (Fig. 4), ERK2 expression in ventral hippocampus was significantly altered, with SNI animals again showing a reduction in ERK expression compared with Sham animals $\left(t_{(6)}=2.95, p=0.025\right)$, indicating a gene expression difference in this brain region in SNI animals (Fig. 4d).

While initially unexpected that changes would be greater in the ipsilateral than contralateral hippocampus, the experiment was replicated numerous times and with surgery alternating on either the left or right hindpaw. In each repetition these results were replicated. Therefore, we conclude that there is distinctive decrease in ERK1 and ERK2 phosphorylation and decrease in ERK2 gene expression levels in SNI animals. It is important to note, that while ERK expression changes are not identical bilaterally, both hemispheres show reduced expression. This decrease in basal ERK expression and phosphorylation could be interfering with the normal action of pERK in contextual fear extinction and influencing the behavioral deficit we observe.

\section{Hippocampal neurogenesis}

Adult hippocampal neurogenesis is indicated to be involved in learning and memory (Deng et al., 2010), and decreases in neurogenesis have been associated with depression (Sahay and Hen, 2007), anxiety (Revest et al., 2009), stress (Dranovsky and Hen, 2006), and impairment in contextual fear conditioning (Saxe et al., 2006). Therefore, we examined the impact of chronic neuropathic pain on hippocampal neurogenesis.

SNI mice showed significantly fewer double-labeled DCX/BrdU cells bilaterally in the subgranular zone of the dentate gyrus of the hippocampus as Sham animals (ipsilateral, $t_{(9)}=5.42$, $p<0.001$; contralateral, $t_{(9)}=4.27, p=$ 0.002 ) indicating a decrease in neurogenesis. SNI mice had an average of 14.4 and 14 cells per hippocampal slice (ipsilateral and contralateral, respectively), while Sham animals had an average of 42.5 cells in ipsilateral and 37.7 cells in contralateral dentate gyrus over corresponding slices (Fig. 5). There were no significant differences in the percentage of double-labeled EGFP/BrdU cells which were also triple-labeled with $\mathrm{NeuN}\left(t_{(8)}=1.66\right.$, n.s. $)$ between SNI $($ mean $=57.66 \%)$ and Sham animals (mean $=47.50 \%$ ). At the time of tissue collection, SNI animals $($ mean $=0.01$ ) had significantly lower VF thresholds on the injured side than Sham animals (mean $=0.35 \mathrm{~g} ; t_{(8)}=3.1, p=0.01$ ).

These results demonstrate that chronic neuropathic pain animals show a bilateral reduction in adult hippocampal neurogenesis measured by the double-labeling of DCX/BrdU. This reduction in newly born cells suggests a mechanism by which learning, memory, contextual extinction, and other behaviors affected by chronic pain are being modulated. There is no change in the percentage of newly born cells that are maturing into neurons, determined by triple-labeling with NeuN, suggesting no difference in maturation speed or atrophy post initial growth of the cells.

\section{Hippocampal dentate gyrus-CA3 fEPSP potentiation}

The MAP/ERK pathway plays a key role in synaptic plasticity, and intriguingly, MAPKs have been found in synaptic terminals (Boggio et al., 2007). In particular, it has been shown that ERK activity affects short-term synaptic plasticity (PTP) through phosphorylation of synapsin in cultured systems (Giachello et al., 2010). We therefore investigated whether the large change observed in ERK signaling and decreased neurogenesis in SNI 
animals may influence short-term synaptic plasticity in the hippocampus of SNI animals at this mossy fiber-CA3 synapse.

Plasticity was induced by four tetanic stimulations ( $1 \mathrm{~s}$ each, $100 \mathrm{~Hz}$ ), and we investigated two parameters: the PTP (within $30 \mathrm{~s}$ from the end of the tetanus) and the kinetics of the intratetanus plasticity. We found that SNI animals differed significantly in both these parameters, and in both ipsilateral and contralateral hippocampi relative to the peripheral nerve injury. Synapses from SNI animals showed a marked use-dependent depression between the first and last of the four tetani (Fig. $6 a, b)$, while in Sham animals no difference was detected; additionally, PTP, when normalized to baseline slope values, was significantly reduced from $2.13 \pm 0.36 \%$ (mean \pm SEM) in ipsilateral hippocampus in Sham animals $(n=7)$ to $1.30 \pm 0.20 \%$ in SNI animals $(n=7, p<0.05$; Fig. $6 d)$. PTP was also significantly reduced from $2.75 \pm$ $0.24 \%(n=6)$ in contralateral hippocampus in Sham animals to $1.46 \pm$ $0.58 \%(n=5, p<0.05$; Fig. $6 d)$ in SNI animals. Therefore, short-term plasticity in the hippocampus of SNI animals is significantly altered, in agreement with the observed differences found in ERK signaling.

\section{Hippocampal volume of human} chronic neuropathic pain patients To this point all of the work presented has been performed in a mouse model of chronic neuropathic pain. If we desire, however, to understand the results in terms of human chronic pain patients we need to investigate the impact of persistent pain on the hippocampus in such patients. We therefore analyzed the hippocampal volume of several types of chronic pain patients and compared these values to a normal subject group. To determine whether there are differences in hippocampal morphology between various types of chronic pain conditions, we included CBP, CRPS, and OA patients in the analysis. Previous studies by Baliki et al. (2011) and Apkarian et al. (2009) indicate that these pain conditions show distinct cortical functional activity patterns and regional gray matter density decreases relative to one another. Thus, here we test whether hippocampal volume is also differentially influenced by distinct chronic pain conditions.

An analysis of covariance indicates that both CBP and CRPS patient groups have significantly less left and right hippocampal volume as compared with normal subjects. For the left hippocampus $\left(F_{(3,122)}=3.79, p=0.01\right), \mathrm{CBP}(t=3.09, p=0.002)$ and CRPS $(t=$ $2.27, p=0.02$ ) patient groups differ significantly from normal subjects through planned comparisons (Fig. 7a) (categorical predictors "group" and "sex," continuous predictors of "age" and "brain volume"). The same is true in the right hippocampus $\left(F_{(3,122)}=2.60\right.$, $p=0.05)$ where CBP $(t=2.09, p=0.04)$ and CRPS $(t=2.44, p=$ 0.02 ) again differ significantly from normal subjects (Fig. $7 b$ ). OA subjects did not differ significantly from normal subjects in hippocampal volume in left $(t=1.09, p=0.28)$ or right $(t=1.25, p=$ 0.21 ) hemispheres. Therefore, there is a reduction in hippocampal volume in certain chronic pain conditions but not in others.

\section{Discussion}

In this study we reveal multiple hippocampal abnormalities in animals with chronic pain and demonstrate a potential underlying mechanism for hippocampal-mediated behavioral abnormalities in these animals through the reduction of hippocampal neurogenesis and altered short-term synaptic plasticity. Moreover, we are the first to demonstrate decreased hippocampal volume in humans with specific chronic pain conditions, which may be a consequence of some of the mechanisms we observe in the rodent model for chronic pain.

Our results demonstrate that SNI and Sham animals learn fear conditioning for context and cue equally well, but only SNI animals are unable to extinguish context fear conditioning. This 
a
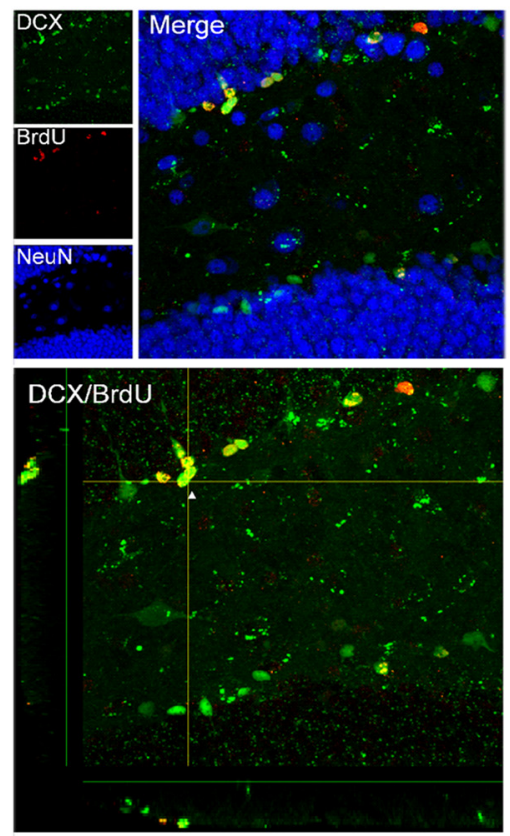

b
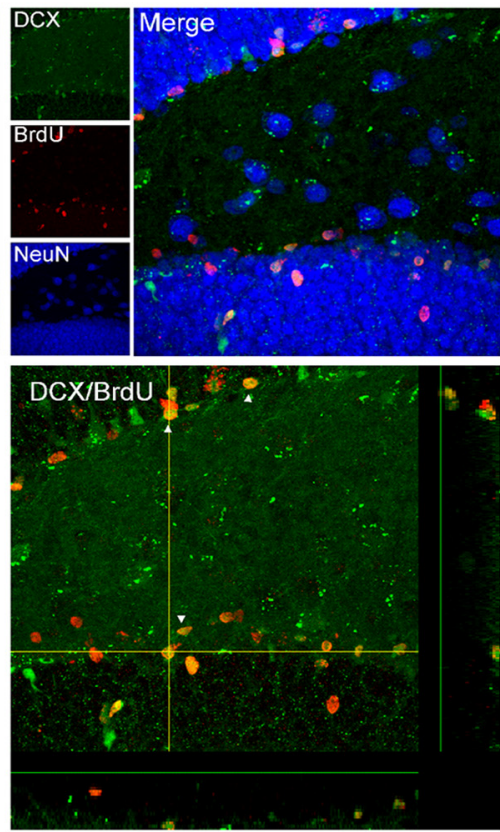

C
DCX/BrdU Positive Cells Ipsilateral Hippocampus

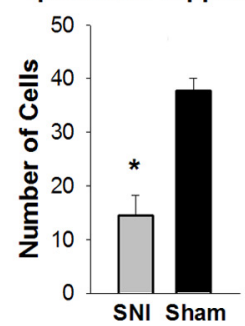

Contralateral Hippocampus

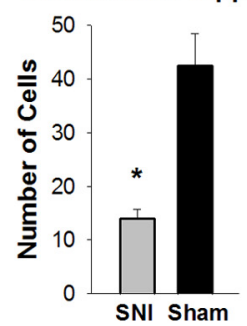

Figure 5. Reduced hippocampal neurogenesis in SNI animals. $a$, Top, Triple-labeling for DCX, BrdU, and NeuN in SNI animals. SNI animals had an average of $57.66 \%$ DCX/BrdU expressing cells which also express NeuN. Bottom, DCX/BrdU double-labeled cells in SNI animals. SNI mice had an average of 14.4 double-labeled cells in the ipsilateral dentate gyrus per hippocampal slice and 14 in the contralateral dentate gyrus. White arrows indicate examples of double-labeling. $\boldsymbol{b}$, Top, Triple-labeling for DCX, BrdU, and NeuN in Sham animals. Sham animals had an average of 47.50\% DCX/BrdU-expressing cells which also express NeuN. This does not differ significantly from SNI animals $\left(t_{(8)}=1.66, n s\right)$. Bottom, DCX/BrdU double-labeled cells in Sham animals. Sham animals had an average of 42.5 cells double-labeled in ipsilateral dentate gyrus and 37.7 double-labeled cells in contralateral dentate gyrus per hippocampal slice. Sham differs significantly from SNI animals both in ipsilateral dentate gyrus $\left(t_{(9)}=5.42, p<0.001\right)$ and contralateral dentate gyrus $\left(t_{(9)}=4.27, p=0.002\right)$. c, Bar graph of number of DCX/BrdU-labeled cells in SNI and Sham animals as described in $\boldsymbol{b}$. Error bars indicate SEM ${ }^{*} p<0.5$. SNI animals had significantly fewer double-labeled cells as Sham animals in both ipsilateral and contralateral hippocampus.

confirms our primary hypothesis that the presence of ongoing pain may interfere particularly with hippocampus-dependent context extinction, while hippocampus-independent cue extinction remains unaffected. The specificity of this deficit points to a modulation of hippocampal functioning, and not to a more general change in fear conditioning circuitry.

SNI animals displayed increased anxiety-like behavior. However, they did not show diminished cue extinction as seen in studies of anxiety disorders (Green et al., 2011) and the ERK expression changes were different from what is seen in studies of anxiety and depression in response to fear conditioning and extinction (Tronson et al., 2008). The bilateral but dissimilar changes may be consistent with previous persistent pain research demonstrating lateralization of ERK changes in the amygdala (Carrasquillo and Gereau, 2007). This suggests that the observed effects are not due to a general stress on the animal, but rather to the presence of pain. Therefore, while this animal model exhibits anxiety-like behavior similar to what is often seen in clinical pain conditions (Gore et al., 2011), it cannot be considered the cause of the observed hippocampal molecular changes and deficits in contextual fear extinction.

The lack of context extinction in SNI animals demonstrates a memory deficit in these animals, which expands and complements previous work that revealed impairments in working and short-term memory in SNI (Ren et al., 2011). While behavioral changes are typically considered linked to long-term synaptic changes, mutant mice lacking synapsin II showed profound learning impairments, which at the cellular level are associated with decreased PTP, without any detectable alteration of long-term plasticity (Silva et al., 1996); intriguingly, such impairments were selective for contextual conditioning, without detectable abnormalities in spatial learning. Previous reports revealed that ERK is present in spines and synaptic terminals (Boggio et al., 2007) and that short-term plasticity is dependent on the MAP/ERK pathway (Giachello et al., 2010). This framework ideally fits our data, which show significant changes in hippocampal ERK expression and impaired short-term plasticity (PTP) in neuropathic animals. Additionally, experiments performed in the calyx of Held, another glutamatergic synapse, demonstrated that deletion of synapsins 1 and 2, which are targets of MAP/ERK phosphorylation, increases the use-dependent synaptic depression induced by a high-frequency stimulus train. This effect was selective for highfrequency trains and was produced through a reduction of the fraction of the readily releasable vesicle pool late in the train (Sun et al., 2006). It is possible that a similar mechanism is at work at the mossy fiber-CA3 synapse. Furthermore, an increase in dentate gyrus granule cells is seen following highfrequency stimulation of the mossy fiber efferents of granule cells (Derrick et al., 2000). This indicates that the changes we observe in synaptic plasticity may tie to neurogenesis and suggests a mechanistic link between the two.

While it is well established that decreases in hippocampal neurogenesis are associated with many comorbid conditions of chronic pain and that this decrease plays an important role in many of the observed cognitive deficits (Dranovsky and Hen, 2006; Revest et al., 2009; Saxe et al., 2006; Sahay and Hen, 2007), only one study has investigated the decrease in neurogenesis in chronic neuropathic pain (Terada et al., 2008). However, these investigators were interested in how chronic neuropathic pain alters enriched-environment increases in neurogenesis. Our study, in contrast, indicates a more direct baseline impact of chronic pain on neurogenesis and not only a modulatory role. Our current work is the first to demonstrate that normal neurogenesis is decreased in the presence of chronic neuropathic pain, which is consistent with the observed abnormal context extinction and altered synaptic plasticity and supports our theory 
that the hippocampal learning and memory system may be critically involved in chronic pain. The mechanism of this reduction in neurogenesis, which we propose then leads to changes in mossy fiber-CA3 plasticity and observed context extinction deficits, is still being explored. It is known, however, that ERK2 is necessary for cell survival (Aouadi et al., 2006), so the reduction of ERK2 observed in the hippocampus of SNI animals may influence the reduction of neurogenic cells.

The reduction in neurogenesis may also involve proinflammatory cytokines, such as TNF- $\alpha$ (Ren et al., 2011), IL-1 $\beta$ (del Rey et al., 2011), or others found to be affected in the hippocampus postperipheral injury (Al-Amin et al., 2011; del Rey et al., 2011). It is suggested by Ren et al. (2011) that overproduction of TNF- $\alpha$ in the hippocampus may contribute to the memory deficits seen in these animals. TNF- $\alpha$ production is stimulated by ERK1/2 activation and is involved in signal transduction (Lee et al., 2011), so this observation is consistent with the changes of hippocampal ERK expression and decreased neurogenesis we observe. Furthermore, TNF- $\alpha$ seems to have an effect on adult hippocampal neurogenesis (Montgomery and Bowers, 2012). IL-1 $\beta$ is another potential cytokine that may underlie the behavioral and neurogenesis changes in SNI animals. Work by del Rey et al. (2011) found that IL-1 $\beta$ is upregulated in the hippocampus post-SNI and this upregulation is correlated with neuropathic pain behavior. IL- $1 \beta$ has been suggested to lead to decreased neurogenesis in the elderly (Kuzumaki et al., 2010) and could play a similar role in neuropathic pain.

Our results in the SNI model tie together in a consistent and complementary fashion. A hippocampal-specific behavioral deficit in context extinction is seen, which has previously been shown to depend on ERK phosphorylation (Fischer et al., 2007) and short-term hippocampal plasticity (Silva et al., 1996). In addition, ERK expression and phosphorylation changes are observed in SNI animals, as well as alterations of short-term synaptic plasticity at the mossy fiber-CA3 synapse. This type of potentiation is known to rely on ERK phosphorylation (Selcher et al., 2003; Giachello et al., 2010) and dentate gyrus neurogenesis (Derrick et al., 2000), which is decreased in SNI animals. Future investigation can determine any functional significance to the bilateral but dissimilar changes in ERK expression. Finally, dentate gyrus neurogenesis, known to be involved in context extinction (Saxe et al., 2006), is closely tied to hippocampal volume (Erickson et al., 2011), which is decreased in human chronic pain
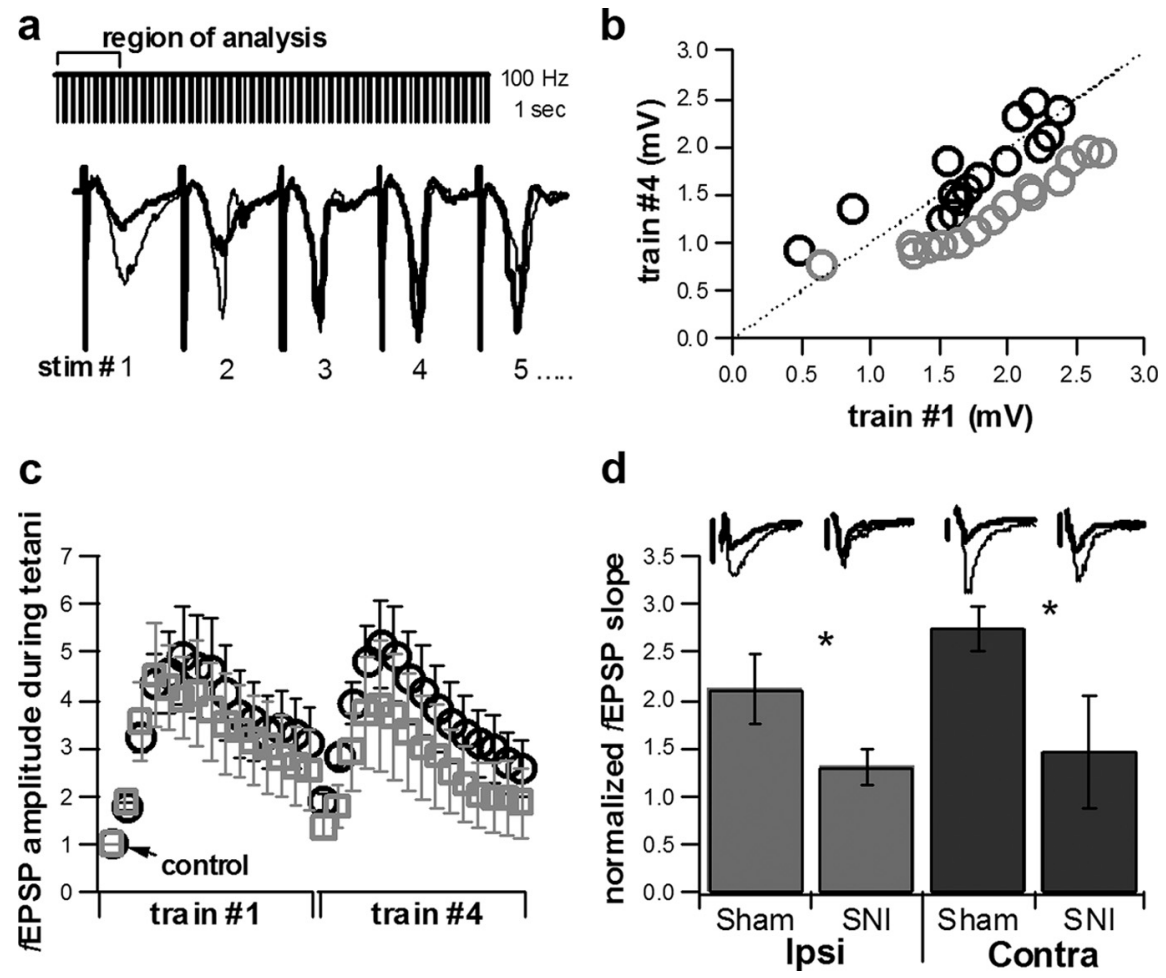

Figure 6. Alterations in short-term plasticity in CA3-mossy fiber pathway in SNI animals. a, Tetanic stimulation ( $1 \mathrm{~s}$ at $100 \mathrm{~Hz}$ 4 trains) in the dentate gyrus. The first 15 fEPSPs of each train were used in the tetanic analysis. Trace showing fEPSPs $1-5$ of train \#1 (bold) versus fEPSPs 1-5 of train \#4 (black). $\boldsymbol{b}$, Linear correlation plot showing average fEPSP amplitude resulting from stim 1 of train \#1 versus stim 1 of train \#4, stim 2 of train \#1 compared with stim 2 of train \#4, etc., in Sham, $n=13$ (black) and SNI, $n=12$ (gray) animals. c, Normalized fEPSP amplitude measurements during tetanic stimulation. The responses to the first 15 stimulations in train \#1 of 4 and the fEPSP responses to the first 15 stimulations of train \#4 of 4 . Results are normalized to the initial response (control). Sham, $n=13$ (black) and SNI, $n=12$ (gray). Error bars indicate SEM.d, Average PTP comparing Sham and SNI animals in both ipsilateral ( $n=7$ and 7, respectively) and contralateral ( $n=6$ and 5, respectively) hemispheres relative to peripheral nerve injury, fEPSP slope following tetanic stimulation normalized to baseline slope measurements. Average normalized PTP exhibited a statistically significant difference when comparing Sham to SNI animals in both hemispheres (one-tailed $t$ test, ${ }^{*} p<0.05$ ). Inset, Traces of Sham and SNI fEPSPs comparing baseline (bold) versus fEPSPs exhibiting PTP. Scale bars: $0.4 \mathrm{mV}$ for all traces except contralateral Sham where the scale bar equals $0.5 \mathrm{mV}$.
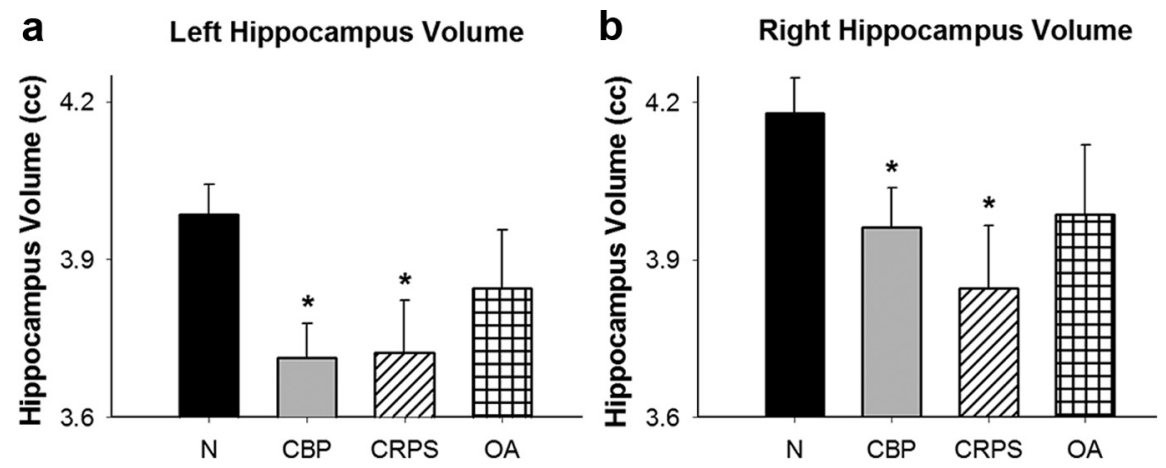

Figure 7. Decreases in hippocampal volume in human chronic pain patients. $\boldsymbol{a}$, Left hippocampal volume of normal subjects (N), CBP, CRPS, and OA. Error bars indicate SEM, ${ }^{*} p<0.05$ from N. CBP $(t=3.09, p=0.002)$ and CRPS $(t=2.27, p=0.02)$ patient groups differ significantly from normal subjects. $\boldsymbol{b}$, Right hippocampal volume of N, CBP, CRPS, and OA groups. CBP $(t=$ $2.09, p=0.04)$ and CRPS $(t=2.44, p=0.02)$ patient groups differ significantly from normal subjects.

patients. Thus, we can presume that the presence of pain per se interferes with mossy fiber-CA3 synaptic plasticity and dentate gyrus neurogenesis. The changes in these hippocampal properties may relate to the hippocampal volume decreases in chronic pain patients.

Until this current study there has been very little data available on the hippocampus and pain patients. A single human study of 
hippocampal volume examined elderly chronic pain patients but observed only a borderline decrease of volume correlated with increased pain (Zimmerman et al., 2009). In our current study we show robust decreases in hippocampus volume in two of the three chronic pain conditions examined. The specificity in these results between patient groups supports the conclusion from previous work that different chronic pain conditions may have unique brain profiles (Apkarian et al., 2009; Baliki et al., 2011). As CBP and CRPS can have a larger neuropathic component than $\mathrm{OA}$, which is regarded as more inflammatory in nature, neuropathic chronic pain conditions seem to have a larger impact on hippocampal volume. Decreased hippocampal volume has been repeatedly observed for depression (Cole et al., 2011), and recently been tied to problems in contextual fear conditioning in humans (Pohlack et al., 2012). Even though human chronic pain is commonly associated with increased depression (Gore et al., 2011), in the current study depression values were not correlated to hippocampal volume and volume decreases in the patients were determined after correcting for depression. Thus, obtained results are due to the presence of chronic pain independent of accompanying depression. This decreased volume may in turn contribute to emotional and cognitive problems seen in chronic pain patients and observed in the present study of an animal chronic pain model.

In summary, we observe robust behavioral, molecular, and synaptic changes in the hippocampus of animals with SNI and, in human chronic pain patients, we show decreased hippocampal volume which may be a consequence of processes similar to those we report in the rodent model of chronic pain. We conclude that these unique hippocampal disruptions in chronic pain cannot be explained by comorbidity with other disorders and that these are specific cellular correlates of the observed cognitive and emotional problems seen both in animal models of chronic pain and human pain patients. Therefore, targeting the reversal of these systematic changes in chronic pain could improve both patient quality of life and actual pain behavior.

\section{References}

Al-Amin H, Sarkis R, Atweh S, Jabbur S, Saadé N (2011) Chronic dizocilpine or apomorphine and development of neuropathy in two animal models II: effects on brain cytokines and neurotrophins. Exp Neurol 228:30-40.

Aouadi M, Binetruy B, Caron L, Le Marchand-Brustel Y, Bost F (2006) Role of MAPKs in development and differentiation: lessons from knockout mice. Biochimie 88:1091-1098.

Apkarian AV (2008) Pain perception in relation to emotional learning. Curr Opin Neurobiol 18:464-468.

Apkarian AV, Sosa Y, Sonty S, Levy RM, Harden RN, Parrish TB, Gitelman DR (2004a) Chronic back pain is associated with decreased prefrontal and thalamic gray matter density. J Neurosci 24:10410-10415.

Apkarian AV, Sosa Y, Krauss BR, Thomas PS, Fredrickson BE, Levy RE, Harden RN, Chialvo DR (2004b) Chronic pain patients are impaired on an emotional decision-making task. Pain 108:129-136.

Apkarian AV, Baliki MN, Geha PY (2009) Towards a theory of chronic pain. Prog Neurobiol 87:81-97.

Apkarian AV, Hashmi JA, Baliki MN (2011) Pain and the brain: specificity and plasticity of the brain in clinical chronic pain. Pain 152:S49-64.

Baliki MN, Chialvo DR, Geha PY, Levy RM, Harden RN, Parrish TB, Apkarian AV (2006) Chronic pain and the emotional brain: specific brain activity associated with spontaneous fluctuations of intensity of chronic back pain. J Neurosci 26:12165-12173.

Baliki MN, Geha PY, Apkarian AV, Chialvo DR (2008) Beyond feeling: chronic pain hurts the brain, disrupting the default-mode network dynamics. J Neurosci 28:1398-1403.

Baliki MN, Schnitzer TJ, Bauer WR, Apkarian AV (2011) Brain morphological signatures for chronic pain. PLoS One 6:e26010.

Barkus C, McHugh SB, Sprengel R, Seeburg PH, Rawlins JN, Bannerman DM
(2010) Hippocampal NMDA receptors and anxiety: at the interface between cognition and emotion. Eur J Pharmacol 626:49-56.

Boggio EM, Putignano E, Sassoè-Pognetto M, Pizzorusso T, Giustetto M (2007) Visual stimulation activates ERK in synaptic and somatic compartments of rat cortical neurons with parallel kinetics. PLoS One 2:e604.

Carrasquillo Y, Gereau RW 4th (2007) Activation of the extracellular signalregulated kinase in the amygdala modulates pain perception. J Neurosci 27:1543-1551.

Chaplan SR, Bach FW, Pogrel JW, Chung JM, Yaksh TL (1994) Quantitative assessment of tactile allodynia in the rat paw. J Neurosci Methods 53:55-63.

Cole J, Costafreda SG, McGuffin P, Fu CH (2011) Hippocampal atrophy in first episode depression: a meta-analysis of magnetic resonance imaging studies. J Affect Disord 134:483-487.

David DJ, Samuels BA, Rainer Q, Wang JW, Marsteller D, Mendez I, Drew M, Craig DA, Guiard BP, Guilloux JP, Artymyshyn RP, Gardier AM, Gerald C, Antonijevic IA, Leonardo ED, Hen R (2009) Neurogenesisdependent and -independent effects of fluoxetine in an animal model of anxiety/depression. Neuron 62:479-493.

Decosterd I, Woolf CJ (2000) Spared nerve injury: an animal model of persistent peripheral neuropathic pain. Pain 87:149-158.

del Rey A, Yau HJ, Randolf A, Centeno MV, Wildmann J, Martina M, Besedovsky HO, Apkarian AV (2011) Chronic neuropathic pain-like behavior correlates with IL- $1 \beta$ expression and disrupts cytokine interactions in the hippocampus. Pain 152:2827-2835.

Deng W, Aimone JB, Gage FH (2010) New neurons and new memories: how does adult hippocampal neurogenesis affect learning and memory? Nat Rev Neurosci 11:339-350.

Derrick BE, York AD, Martinez JL Jr (2000) Increased granule cell neurogenesis in the adult dentate gyrus following mossy fiber stimulation sufficient to induce long-term potentiation. Brain Res 857:300-307.

Dick BD, Rashiq S (2007) Disruption of attention and working memory traces in individuals with chronic pain. Anesth Analg 104:1223-1229, tables of contents.

Dranovsky A, Hen R (2006) Hippocampal neurogenesis: regulation by stress and antidepressants. Biol Psychiatry 59:1136-1143.

Erickson KI, Voss MW, Prakash RS, Basak C, Szabo A, Chaddock L, Kim JS, Heo S, Alves H, White SM, Wojcicki TR, Mailey E, Vieira VJ, Martin SA, Pence BD, Woods JA, McAuley E, Kramer AF (2011) Exercise training increases size of hippocampus and improves memory. Proc Natl Acad Sci U S A 108:3017-3022.

Fischer A, Radulovic M, Schrick C, Sananbenesi F, Godovac-Zimmermann J, Radulovic J (2007) Hippocampal Mek/ERK signaling mediates extinction of contextual freezing behavior. Neurobiol Learn Mem 87:149-158.

Flor H, Knost B, Birbaumer N (2002) The role of operant conditioning in chronic pain: an experimental investigation. Pain 95:111-118.

Giachello CN, Fiumara F, Giacomini C, Corradi A, Milanese C, Ghirardi M, Benfenati F, Montarolo PG (2010) MAPK/ERK-dependent phosphorylation of synapsin mediates formation of functional synapses and shortterm homosynaptic plasticity. J Cell Sci 123:881-893.

Gore M, Sadosky A, Stacey BR, Tai KS, Leslie D (2011) The burden of chronic low back pain: clinical comorbidities, treatment patterns, and healthcare costs in usual care settings. Spine [Epub ahead of print].

Green MK, Rani CS, Joshi A, Soto-Piña AE, Martinez PA, Frazer A, Strong R, Morilak DA (2011) Prenatal stress induces long term stress vulnerability, compromising stress response systems in the brain and impairing extinction of conditioned fear after adult stress. Neuroscience 192:438-451.

Han JS, Neugebauer V (2005) mGluR1 and mGluR5 antagonists in the amygdala inhibit different components of audible and ultrasonic vocalizations in a model of arthritic pain. Pain 113:211-222.

Ji G, Sun H, Fu Y, Li Z, Pais-Vieira M, Galhardo V, Neugebauer V (2010) Cognitive impairment in pain through amygdala-driven prefrontal cortical deactivation. J Neurosci 30:5451-5464.

Kishimoto T, Radulovic J, Radulovic M, Lin CR, Schrick C, Hooshmand F, Hermanson O, Rosenfeld MG, Spiess J (2000) Deletion of crhr2 reveals an anxiolytic role for corticotropin-releasing hormone receptor-2. Nat Genet 24:415-419.

Kodama D, Ono H, Tanabe M (2007) Altered hippocampal long-term potentiation after peripheral nerve injury in mice. Eur J Pharmacol 574:127-132.

Kodama D, Ono H, Tanabe M (2011) Increased hippocampal glycine 
uptake and cognitive dysfunction after peripheral nerve injury. Pain 152:809-817.

Kuzumaki N, Ikegami D, Imai S, Narita M, Tamura R, Yajima M, Suzuki A, Miyashita K, Niikura K, Takeshima H, Ando T, Ushijima T, Suzuki T, Narita M (2010) Enhanced IL-1beta production in response to the activation of hippocampal glial cells impairs neurogenesis in aged mice. Synapse 64:721-728.

Lee EO, Kim SE, Park HK, Kang JL, Chong YH (2011) Extracellular HIV-1 Tat upregulates TNF-alpha dependent MCP-1/CCL2 production via activation of ERK1/2 pathway in rat hippocampal slice cultures: inhibition by resveratrol, a polyphenolic phytostilbene. Exp Neurol 229:399-408.

Li XY, Ko HG, Chen T, Descalzi G, Koga K, Wang H, Kim SS, Shang Y, Kwak C, Park SW, Shim J, Lee K, Collingridge GL, Kaang BK, Zhuo M (2010) Alleviating neuropathic pain hypersensitivity by inhibiting PKMzeta in the anterior cingulate cortex. Science 330:1400-1404.

Metz AE, Yau HJ, Centeno MV, Apkarian AV, Martina M (2009) Morphological and functional reorganization of rat medial prefrontal cortex in neuropathic pain. Proc Natl Acad Sci U S A 106:2423-2428.

Montgomery SL, Bowers WJ (2012) Tumor necrosis factor-alpha and the roles it plays in homeostatic and degenerative processes within the central nervous system. J Neuroimmune Pharmacol 7:42-59.

Patenaude B (2007) Bayesian statistical models of shape and appearance for subcortical brain segmentation. $\mathrm{PhD}$ thesis, University of Oxford.

Phillips RG, LeDoux JE (1992) Differential contribution of amygdala and hippocampus to cued and contextual fear conditioning. Behav Neurosci 106:274-285.

Pohlack ST, Nees F, Liebscher C, Cacciaglia R, Diener SJ, Ridder S, Woermann FG, Flor H (2012) Hippocampal but not amygdalar volume affects contextual fear conditioning in humans. Hum Brain Mapp $33: 478-488$.

Radulovic J, Rühmann A, Liepold T, Spiess J (1999) Modulation of learning and anxiety by corticotropin-releasing factor (CRF) and stress: differential roles of CRF receptors 1 and 2. J Neurosci 19:5016-5025.

Ren WJ, Liu Y, Zhou LJ, Li W, Zhong Y, Pang RP, Xin WJ, Wei XH, Wang J, Zhu HQ, Wu CY, Qin ZH, Liu G, Liu XG (2011) Peripheral nerve injury leads to working memory deficits and dysfunction of the hippocampus by upregulation of TNF- $\alpha$ in rodents. Neuropsychopharmacology 36:979-992.

Revest JM, Dupret D, Koehl M, Funk-Reiter C, Grosjean N, Piazza PV, Abrous DN (2009) Adult hippocampal neurogenesis is involved in anxiety-related behaviors. Mol Psychiatry 14:959-967.
Sahay A, Hen R (2007) Adult hippocampal neurogenesis in depression. Nat Neurosci 10:1110-1115.

Santarelli L, Saxe M, Gross C, Surget A, Battaglia F, Dulawa S, Weisstaub N, Lee J, Duman R, Arancio O, Belzung C, Hen R (2003) Requirement of hippocampal neurogenesis for the behavioral effects of antidepressants. Science 301:805-809.

Saxe MD, Battaglia F, Wang JW, Malleret G, David DJ, Monckton JE, Garcia AD, Sofroniew MV, Kandel ER, Santarelli L, Hen R, Drew MR (2006) Ablation of hippocampal neurogenesis impairs contextual fear conditioning and synaptic plasticity in the dentate gyrus. Proc Natl Acad Sci U S A 103:17501-17506.

Selcher JC, Weeber EJ, Christian J, Nekrasova T, Landreth GE, Sweatt JD (2003) A role for ERK MAP kinase in physiologic temporal integration in hippocampal area CA1. Learn Mem 10:26-39.

Silva AJ, Rosahl TW, Chapman PF, Marowitz Z, Friedman E, Frankland PW, Cestari V, Cioffi D, Südhof TC, Bourtchuladze R (1996) Impaired learning in mice with abnormal short-lived plasticity. Curr Biol 6:1509-1518.

Smith SM, Jenkinson M, Woolrich MW, Beckmann CF, Behrens TE, Johansen-Berg H, Bannister PR, De Luca M, Drobnjak I, Flitney DE, Niazy RK, Saunders J, Vickers J, Zhang Y, De Stefano N, Brady JM, Matthews PM (2004) Advances in functional and structural MR image analysis and implementation as FSL. Neuroimage 23 [Suppl 1]:S208-S219.

Sun J, Bronk P, Liu X, Han W, Südhof TC (2006) Synapsins regulate usedependent synaptic plasticity in the calyx of Held by a Ca2+/calmodulindependent pathway. Proc Natl Acad Sci U S A 103:2880-2885.

Takahashi LK, Kalin NH, Vanden Burgt JA, Sherman JE (1989) Corticotropin-releasing factor modulates defensive-withdrawal and exploratory behavior in rats. Behav Neurosci 103:648-654.

Terada M, Kuzumaki N, Hareyama N, Imai S, Niikura K, Narita M, Yamazaki M, Suzuki T, Narita M (2008) Suppression of enriched environmentinduced neurogenesis in a rodent model of neuropathic pain. Neurosci Lett 440:314-318.

Tronson NC, Schrick C, Fischer A, Sananbenesi F, Pagès G, Pouysségur J, Radulovic J (2008) Regulatory mechanisms of fear extinction and depression-like behavior. Neuropsychopharmacology 33:1570-1583.

Turner SG, Sechzer JA, Liebelt RA (1967) Sensitivity to electric shock after ventromedial hypothalamic lesions. Exp Neurol 19:236-244.

Zimmerman ME, Pan JW, Hetherington HP, Lipton ML, Baigi K, Lipton RB (2009) Hippocampal correlates of pain in healthy elderly adults: a pilot study. Neurology 73:1567-1570. 